\title{
Mongo DB GUI Operation Using Python
}

\author{
Sarkarsinha H. Rajput ${ }^{1}$, Anand S. Jain ${ }^{2}$, Priyanka B. Patil ${ }^{3}$, Mahesh D. Patil ${ }^{4}$ \\ Department of Information Technology, Shram Sadhna Bombay Trust COET, jalgaon, India ${ }^{1,2,3,4}$
}

\begin{abstract}
Mongo DB is a perfect NoSQL database. We can improve performance and index operations speed using this Mongo DB database. There are various features of Mongo DB like increased speed of database operations, various functions instead of complex SQL queries and a very huge performance improvement.

Python is web scripting language used for high usage websites for improving performance of the website and reducing the overhead on the web server. We will use this Python language in integration with Mongo DB for highest possible performance.
\end{abstract}

Keywords: MongoDB, Python, Add data, Delete Data, Update Data, View Data, NoSql.

\section{INTRODUCTION}

"Mongo DB wasn't designed in a lab. Mongo DB is 5. Delete data in the table Open-source database introduced by $10 \mathrm{gen}$, for wide 6 . Select data from table Variety of application.It is an agile database that allow using regular expression Schema to change quick as application evolve. Mongo using constant data DB is High performance, scalability and availability in using multiple data items memory computing. Mongo DB native replication and 7. User and Roles Management Automated failure's enable enterprise grade reliability and 8. Query Statistics also have operational flexibility. Mongo DB is a database which has document oriented storage. It is also object oriented written in $\mathrm{C}++$.

Mongo DB is open-source full index support database. It is easy to use and in the Mongo DB written query is very simple. Mongo DB provide Map Reduce function .It also provide Grid Fs for process on Big Data. Mongo DB is very powerful much flexible and provide very nice speed. Mongo DB is the primary daemon process for Mongo DB system in mongo DB database is physical container for collection. Each database get its own set of file on the file system. A single Mongo DB server typically has multipledatabases.

In Mongo DB collection is Table and document is row And column/attribute/variable is filed. In Mongo DB document is set of key value pair. Mongo DB support Various driverssuch as AggregationFramework. AutoSharading, JSON data model with dynamic schema.

\section{Proposed System}

We are developing the reliable and consistence Interface for Mongo DB.

We will calculate Database operation statistics along with the basic database operation.

Mongo DB not having any proper GUI or easiness in the operations. The procedures for Performing any operations are very tedious. We are trying to keep the operations very Easy, and trying to improve performance of the operations. So that our GUI can be Easily operational and have very high performance.

We will perform following operations in our project:

1. Create database

2. Create table

3. Insert data in the table

4. Update data in the table

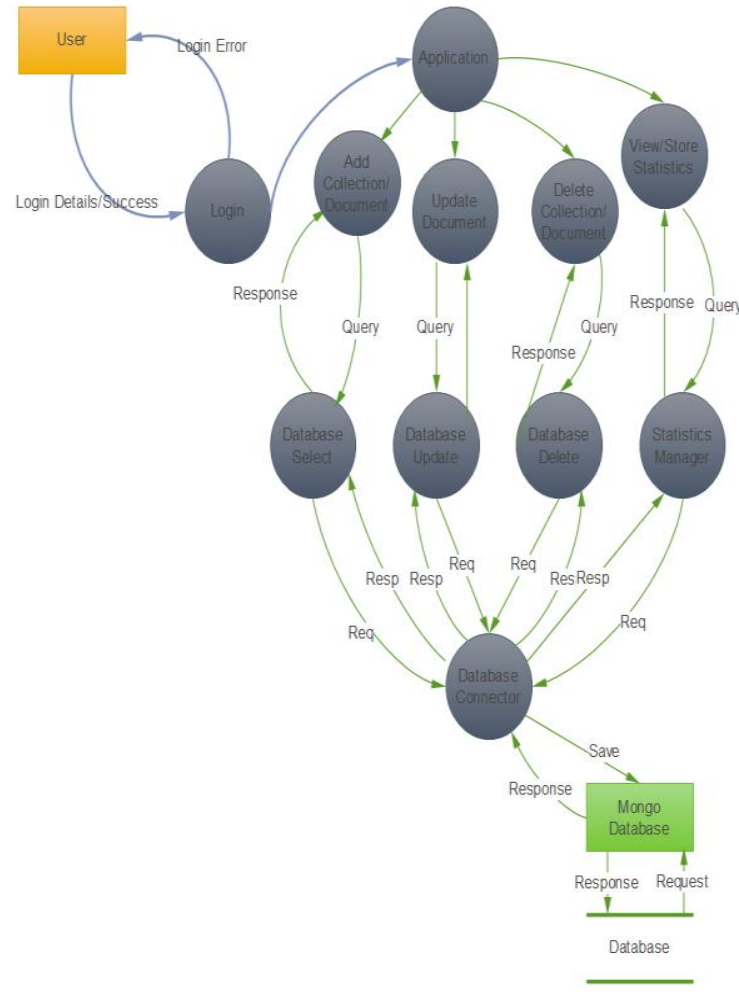

Fig. 1 Architecture of Mongo DB GUI Operation Using Python.

\section{III.FRAMEWORK}

As shown in the figure first we have to login in the Application. After login we can add document, delete Collection or view statistical data. 


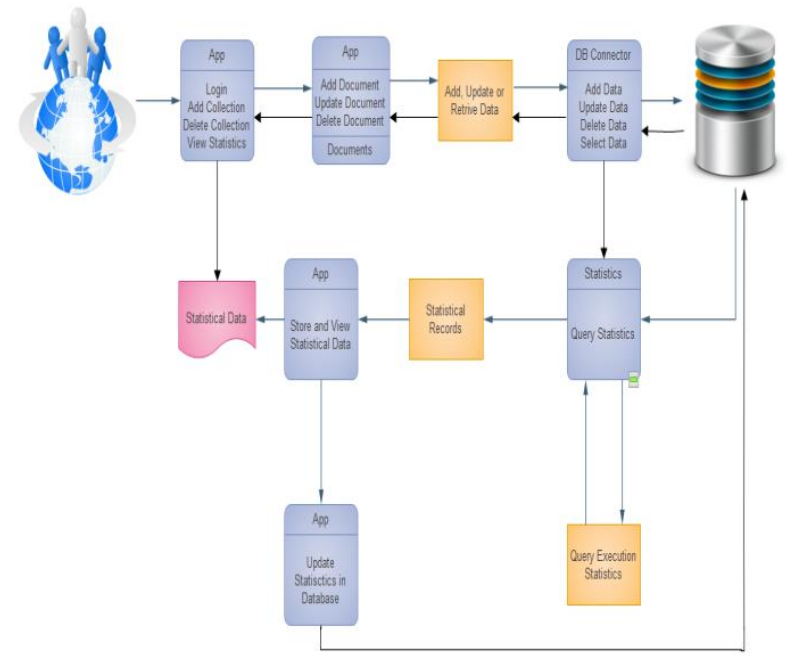

Fig. 2Frame work for Mongo DB GUI Operation Using Python.

\section{A.Steps for Mongo DBDatabase GUI Operation Using} Python.

- $\quad$ Mongo DB does not have proper GUI for client.It use mongo shell but Monngoshell is not user friendlythere four we developed GUI using python with the help of this application user can easy to interact with Mongo DB database

- In this framework first client Login in the application and create database. If user or client is authorized then he or she Login successfully otherwise it has been given a message authentication is failed.

- If authorize user is Login and its create database successfully then its create table

- $\quad$ After creating table user can add, delete, update data in the table.

- In that framework also we can select data in tableusing RegularExpression, using constant data using multiple data items

- $\quad$ After all this process we can manage user roles and find query static.

In such manner we are developing the reliable and consistence Interface for Mongo DB.

We will calculate Database operation statistics along with the basic database operation.. We are trying to keep the operations very easy, and trying to improve performance of the operations. So that our GUI can be Easily operational and have very high performance.

\section{IV.CONCLUSION}

RDBMS won't go away, they're still definitely needed. But the storage requirement for the new generation of applications are huge different from legacy applications. We can choose Mongo DB instead of MySQL because of two factors, ease of use and performance [4].The end user will get a user friendly GUI to interact with Mongo DB. It will perform the Queries in less time than SQL does And it is not replacement for RDBMS but compliment for it. After completing the development of project we have plan to keep it open source. so that other user can contribute to it. And based upon this analysis and design part, we are now able to develop the application

\section{REFERENCES}

[1] R. A. Yogesh Punia, Paper:- implementing information system using mongodb and redis," March 2014

[2] S. S. Kulkarni, M. G. Gouda, and A. Arora, ISecret instantiation in ad-hoc networks,"Computer Communications, vol. 29, no. 2, pp. $200\{215,2006$.

[3] C. K.Wong, M. Gouda, and S. S. Lam, ISecure group communications using key graphs," SIGCOMM Comput. Commun. Rev, vol. 28, no. 4, pp. $68\{79,1998\}$.

[4] Sanobar Khan, Prof.Vanita Mane, IPaper:- "SQL Support over Mongo DB using Metadata,"International Journal of Scientific and Research Publications, Volume 3, Issue 10, October 20131 ISSN 2250-3153.

[5] http://www.mongodb.org/ 